\title{
LOS EFECTOS DE LOS PROGRAMAS PEDAGÓGICOS CURRICULARES QUE APLICAN JUEGOS MOTRICES EN EL DESARROLLO DE LAS FUNCIONES EJECUTIVAS EN ETAPA PREESCOLAR: UNA REVISIÓN SISTEMÁTICA.
}

\author{
THE EFFECTS OF CURRICULAR \\ PEDAGOGICAL PROGRAMS THAT \\ APPLY MOTOR GAMES ON THE \\ DEVELOPMENT OF EXECUTIVE \\ FUNCTIONS IN PRESCHOOL: A \\ SYSTEMATIC REVIEW.
}

\author{
Diana Milena Bedoya Salazar ${ }^{1}$ \\ Alexis Arenas Hoyos ${ }^{2}$ \\ María Elisa Álvarez Sossa ${ }^{3}$ \\ Unidad Central del Valle del Cauca
}

\footnotetext{
1 Investigadora principal: Diana Milena Bedoya Salazar Orcid: https://orcid.org/0000-0002-3363-9797 Correo electrónico: dbedoya@uceva.edu.co Pregrado: Licenciada en Educación Física, Recreación y Deporte (UCEVA). Postgrado: Especialista en Teoría y Metodología del entrenamiento deportivo (Escuela Nacional del Deporte). Docente de la Institución Universitaria Unidad Central del Valle del Cauca (UCEVA) $2 \quad$ Alexis Arenas Hoyos Orcid: https://orcid.org/0000-0002-7933-8498 Licenciado en educación básica, con énfasis en educación física, recreación y deporte (UCEVA) Correo electrónico: alexis.arenas01@uceva.edu.co

$3 \quad$ María Elisa Álvarez Ossa. Doctora en Ciencias de la Educación. RUDECOLOMBIAdocente de la Institución Universitaria. Unidad Central del Valle del Cauca UCEVA,

Líder Grupo de Investigación Educación y Currículo. Correo electrónico: mealvarez@uceva.edu.co. ORCID ID. 0000-0002-4140-7793
} 


\section{RESUMEN}

La presente revisión sistemática tiene como objetivo, describir los efectos de los programas pedagógicos curriculares que aplica juegos motrices en el desarrollo de las funciones ejecutivas durante la etapa preescolar, ya que, el juego motor se ha proclamado como un conductor para el desarrollo cognitivo y ejecutivo, tomando más importancia en edades infantiles, sin embargo, aún quedan vacíos sobre la eficacia en investigaciones de tipo explicativo que toma esta actividad en referencia a los procesos biológicos de neuromaduración y su incidencia en los procesos de aprendizaje durante la etapa preescolar en las instituciones educativas, Los estudios elegibles fueron publicaciones en inglés, portugués y español, revisadas por pares que tomaron muestras de una población de niños con una edad promedio entre 3 y 7 años y se centró en desarrollar y evaluar una función ejecutiva. Los elementos de informe siguieron la guía de revisiones (PRISMA), para finalmente seleccionar nueve investigaciones que cumplieron con los criterios de inclusión. Como resultado, se puede afirmar que el juego motriz y las FE están estrechamente correlacionadas, teniendo una incidencia significativa en la memoria de trabajo, la flexibilidad cognitiva, el control inhibitorio y la planificación y, que estás trascienden en conductas y comportamiento en contextos educativos, sociales, emocionales y principalmente cognitivos, además, los hallazgos evidencian la necesidad de implementar planes de estudio fundados en el juego motor como medio de enseñanza- aprendizaje en etapas preescolares, puesto que presenta mayor beneficio en los procesos de neuroplasticidad y aprendizaje.

PALABRAS CLAVES: funciones ejecutivas, preescolar, juego motriz, desarrollo.

\section{ABSTRACT}

The purpose of this systematic review is to describe the effects of curricular pedagogical programs that apply motor games in the development of executive functions during the preschool stage, since motor games have been proclaimed as a driver for cognitive and executive development, taking more importance in infantile ages, however, there are still gaps on the effectiveness of explanatory research on this activity in reference to the biological processes of neuromaturation and its impact on learning processes during the preschool stage in educational institutions, The eligible studies were peer-reviewed publications in English, Portuguese and Spanish that sampled a population of children with an average age between 3 and 7 years old and focused on developing and assessing an executive function. The reporting items followed the review guide (PRISMA), to finally select nine investigations that met the inclusion criteria. As a result, it can be affirmed that motor play and EF are closely correlated, having a significant impact on working memory, cognitive flexibility, inhibitory control and planning, and that these transcend in behaviors and behavior in educational, social, emotional and mainly cognitive contexts. In addition, the findings show the need to implement study plans based on motor play as a means of teaching-learning in preschool stages, since it presents greater benefit in the processes of neuroplasticity and learning.

KEY WORDS: executive functions, preschool, motor play, development.

\section{INTRODUCCIÓN}

La etapa preescolar se caracteriza por un intenso deseo por el movimiento y el juego, una evidente curiosidad por todo lo desconocido, el gusto por la invención y la predisposición afectiva hacia el aprendizaje, por medio de esta actividad, expresan sus emociones y su espontaneidad, agregando la posibilidad de explorar el entorno gracias a las interacciones con el mismo y 
con los demás, dando de este modo, origen a procesos cognitivos (Guillen, 2017; Mora, 2017).

Del mismo modo, se han conocido los beneficios de construir aprendizajes fundados en la educación del movimiento (Bartholomew, 2017; Goh, 2017), los estudios demuestran su influencia y correlación con el desarrollo cognitivo, comenzando con la facilidad de crear mayor cantidad de conexiones neurales y sinápticas entre diferentes regiones del cerebro como la corteza cerebral, el hipocampo y la amígdala (sistema límbico), y mayor activación en el cuerpo calloso, lo que repercute en la activación de los hemisferios (Bueno, 2019; Meinel \& Schnabel, 2004;Vidarte \& Orozco, 2015). Igualmente, si la estimulación va acompañada de una experiencia motivacional, emocional y de experiencias significativas de aprendizaje, las conexiones sinápticas que se crean serán mayores y duraderas, pues se sustenta, que, al existir una relación entre regulación cognitiva y emocional, los niños y niñas tendrán un mejor ajuste escolar y social (Traverso, 2015; Mora, 2017).

Asimismo, la motricidad tiene una relación directa con la coordinación, esto se puede entender desde la regulación de un acto motor, que a su vez debe ser calculado y regulado conscientemente para lograr un objetivo (Weineck, 2005) es decir, una acción motriz debe ser conducida, planeada y ajustada en la base de sistemas regulativos sensomotrices, en procesos fisiológicos y bioquímicos y sobre las habilidades cognitivas, implicando una acción sobre el sistema nervioso central, que crea consciencia sensorial acerca de los movimientos (Bueno, 2019; Rigal, 2006). Por ejemplo, el control postural, la estructuración espaciotiempo, la velocidad y la lateralidad están relacionados directamente con la motricidad y a su vez con la coordinación, dicho de otro modo, el cerebro posee una mayor plasticidad neural durante edades tempranas, siendo una etapa sensible de progreso motor, que representará más facilidad en las conexiones sinápticas, que a su vez favorecerá un adecuado desarrollo cognitivo, emocional, y social, representado en un mejor aprendizaje en contextos escolares (Meinel \& Schnabel, 2004; Vidarte y Orozco, 2015).

Igualmente, Piaget (1956) ostenta que las habilidades cognitivas están fundamentadas en la motricidad, esto quiere decir que la inteligencia es resultado de las experiencias motrices que pueden recibir del entorno, sin embargo Parlebas, (2008) propone recentrar esta premisa, entendiendo que un niño que salta, juega y corre, está descifrando, decodificando y elaborando respuestas de acción, que a su vez, constituyen y desarrollan los mecanismos cognitivos, deduciendo procesos posteriores más elaborados que serán la base de la inteligencia.

Ahora bien, las FE deben ser atizadas en ámbitos educativos; una de las formas más efectivas para desplegar su función, es por medio del juego motor, ya que favorece los procesos psicosociales y los rasgos característicos de la personalidad del niño, como lo expresa Blair \& Raver (2015), el desarrollo de las FE depende de la maduración biológica de las estructuras anteriores del cerebro, pero además, de los estímulos derivados de actividades con un nivel apropiado de complejidad que presenten las interacciones $\mathrm{e}$ integraciones entre la neocorteza y el sistema límbico, produciendo mayores conexiones sinápticas. Todos estos procesos necesitan actividad física y mental para un óptimo progreso de las FE. Por ejemplo, al ejecutar una acción motriz los niños y niñas deben secuenciar movimientos que permitan alcanzar el objetivo de aprendizaje de ese juego, es decir, ellos lo interiorizan, analizando las posibilidades de ejecución y al final lo expresan con un patrón de movimiento (Barkley, 2011; 
Johnson et al, 1987; Sarlé, 2016; Stein et al, 2012; Vygotsky, 1995).

En este sentido y de acuerdo a las teorías, los juegos motores son los medios más transversales en el desarrollo de habilidades cognitivas, emocionales y sociales para niños y jóvenes, por lo que, aquellos aprendizajes construidos sobre un óptimo desarrollo motor, puede influir en la adquisición de aprendizajes posteriores más complejos, como la lecto-escritura o la aritmética y un adecuado funcionamiento ejecutivo (Cameron et al. 2012; Lundy \& Trawick Smith, 2020; Yogman, et. al, 2018).

En este sentido, se ha expresado en los apartados anteriores la relación del juego motor, las FE y su interdependencia en el desarrollo de las habilidades cognitivas en etapas preescolares, sin embargo, es necesario ahondar en los fundamentos que demuestran tales argumentos, por lo cual, se plantea la problemática y es preciso describir: ¿Cuáles son los efectos de los programas pedagógicos curriculares que aplican juegos motrices en el desarrollo de las funciones ejecutivas durante la etapa preescolar? para lo cual, se busca evidenciar en las teorías existentes basados en la investigación científica; la incidencia producida a nivel cognitivo y funcionamiento ejecutivo, ya que, casi siempre, se encuentra información en estudios de tipo descriptivo, siendo imprescindible corroborar a profundidad los cambios producidos en estudios de tipo explicativo de corte longitudinal y con intervenciones realizadas a esta población, documentando la aplicación de programas curriculares que usan medios y métodos asociados a los juegos motrices como medio consolidación de las FE, paralelas a la etapa de crecimiento y neuromaduración en niños y niñas en etapa preescolar.

\section{FUNCIONES EJECUTIVAS}

Respecto a las FE cabe aclarar, que no existe un consenso en su conceptualización, sin embargo, autores como Braidot, (2015); Bueno, (2019); Gonzáles, (2014); Zelazo et al, (2008) las definen como un término paraguas que agrupa diferentes habilidades cognitivas de orden superior e inferior, entre ellas, la autorregulación, control inhibitorio, planificación, flexibilidad cognitiva, memoria, atención, entre otros. Asimismo, el término FE, se describe cómo los procesos de autorregulación de orden superior que ayudan a supervisar y controlar el pensamiento y la acción (Zelazo et al. 2008). De esta forma, el concepto de FE contiene diversas habilidades específicas que se relacionan entre sí, dentro de las que destacan: el Control Inhibitorio $(\mathrm{Cl})$, entendida como la capacidad controlar las respuestas impulsivas utilizando la atención y el razonamiento para responder, la Memoria de Trabajo (MT) como la capacidad de recordar y utilizar información relevante durante una actividad y la Flexibilidad Cognitiva (FC) es desentenderse de un conjunto de tareas irrelevantes y tomar decisiones convenientes (Sunyer et al. 2015; Diamond, 2016).

Como analogía, las FE desempeñan un rol dirigente en diferentes procesos cognitivos, como lo son actividades mentales complejas, necesarias para planificar, organizar, guiar, revisar, regularizar y evaluar el comportamiento necesario para adaptarse eficazmente al entorno y para alcanzar metas (Bauermeister, 2008). Aunque la mayor parte de la actividad cerebral se produce de forma preconsciente, en la activación de sus redes neuronales residen características tan complejas como la consciencia, la capacidad de tomar decisiones de forma reflexiva, el razonamiento y el control voluntario del pensamiento, en este aspecto, las FE son un constructo que agrupan habilidades cognitivas más complicadas, como lo expone Jin Chung et al. (2014) refiriéndose específicamente a las habilidades cognitivas que permiten la planificación o formulación de objetivos dirigido a metas, autorregulación de las emociones, respuestas adaptativas o toma de decisiones 
frente a una actividad dirigida a la solución de problemas, en situaciones nuevas y complejas. Dicho lo anterior, es fundamental determinar la importancia que tiene el juego motor en el desarrollo de las FE, siendo el principal propósito de esta revisión.

Del mismo modo, Diamond \& Ling (2016); Ramos et al. (2019) comprobaron que las FE pueden ser entrenadas y mejoradas a cualquier edad, mediante el entrenamiento y la práctica, usando distintos métodos y actividades de estimulación cognitiva, como los videojuegos, la música, la actividad física, las artes marciales, el teatro y planes de estudios escolares; se infirió que las intervenciones con enfoques que involucran aspectos emocionales, sociales y físicos tuvieron más éxito que los recursos computarizados, en síntesis, un adecuado funcionamiento ejecutivo puede predecir la calidad de vida, la salud mental y los logros académicos.

\section{NEUROMADURACIÓN DE LAS FUNCIONES EJECUTIVAS EN ETAPA PREESCOLAR:}

En cuanto a la consolidación de las FE, Muchiut (2019) menciona que durante la etapa preescolar, los niños generan procesos madurativos del sistema nervioso central permitiendo el desarrollo de las habilidades cognitivas básicas produciendo diferentes procesos neuropsicológicos como la atención, la memoria y las FE, del mismo modo, Bausela (2014) menciona, que durante el crecimiento y maduración biológica de los niños y las niñas, se desarrollan habilidades cognitivas como la planificación y toma de decisiones, pero estas deben ser potenciadas y estimuladas acordes a las necesidades de aprendizaje.

En este sentido, el desarrollo de las FE durante la infancia y etapa preescolar sufre algunos cambios. Según Merino, (2016) el cerebro reptiliano es predominante en la conducta del niño a partir del primer año, buscando satisfacer sus necesidades básicas de supervivencia y de bienestar. De los cero a los tres años, se inicia la maduración de las estructuras subcorticales, incluyendo el sistema límbico (tálamo, hipocampo, amígdalas) permitiendo un aprendizaje instintivo en la necesidad de explorar y conocer las posibilidades y riesgos del entorno a partir de la observación, la percepción y la manipulación (Bueno, 2019), en este momento, se trata de conectar con el sistema instintivo emocional del niño a través de los límites, las normas, el afecto y la presencia.

El cerebro racional y la conducta cobra cada vez más protagonismo en la vida del niño a partir del tercer año, algunos estudios demuestran que a los cinco años, ya se han desarrollado parcialmente algunas FE como son la memoria de trabajo, la inhibición y la flexibilidad cognitiva, igualmente, en un estudio realizado por Moriguchi \& Hiraki, (2011) se pudo corroborar el desarrollo de la corteza prefrontal durante los tres y cuatro años, examinando la activación prefrontal usando espectroscopia de infrarrojo cercano, evidenciando la activación prefrontal en tareas específicas, lo que demostró un desarrollo cognitivo asociado a la edad de crecimiento. En concordancia, los estudios de neuroimagen funcional en preescolares muestran que las áreas de la corteza prefrontal se muestran activas durante tareas de FE, por ejemplo, Tsujimoto y otros (2004) examinaron la activación de la región corteza prefrontal durante tareas de memoria de trabajo en niños de cinco y seis años, encontraron que los patrones de activación fueron similares a los de los adultos que también participaron en dicho estudio.

En el mismo orden de ideas, distintos estudios que utilizan diversas medidas de control inhibitorio en niños, han sugerido que las FE pueden desempeñar un papel importante en el desarrollo cognitivo y social, como la teoría de la mente, el juego, la competencia social, la conducta moral y la preparación escolar, por el contrario, las alteraciones de las FE están 
implicadas en varios trastornos infantiles, poco control cognitivo, dificultad para mantenerse en una tarea prolongada, problemas para tomar decisiones, asociados a un mal ajuste social y académico (Zelazo, et al. 2008).

Por otro lado, González Osornio (2014) aseveran que existe un proceso de neuromaduración de las FE en los niños preescolares que van aumentando en complejidad a medida que crecen con la edad, asimismo, se reconoce que a edades tempranas ( 3 a 6 años), las FE dependen de la unidad de dos componentes: memoria de trabajo e inhibición, los cuales participan de manera independiente, pero también se relacionan con otras áreas neuronales. Es decir, la unidad estructural de las FE en edades muy tempranas, cambia a través del desarrollo, siendo cada vez más multifacética, que se relaciona con la maduración e integración de diferentes circuitos frontosubcorticales.

\section{CLASIFICACIÓN DE JUEGOS MOTORES.}

Acerca del juego, se puede afirmar que hace parte del desarrollo del ser humano desde la antigüedad, desde su nacimiento, el individuo tiene incorporado esta actividad y gracias a este, el menor se relaciona con su entorno. Es considerado por algunos autores como una actividad voluntaria y natural en los niños, donde ellos expresan sus emociones; se desarrolla bajo parametros, como tiempo, lugar y reglas (Diaz, 1993; Huizinga, 1990). Hoy en día, el juego es utilizado como un conductor hacia el aprendizaje, aún más en etapas sensibles donde los estímulos producidos por el juego generan un papel fundamental para el desarrollo del menor,

Se ha dicho que el juego hace parte fundamental en el crecimiento y la educación de los niños y niñas. Los estímulos derivados mantienen su atención y motivación en la necesidad de establecer conexiones con su entorno de una forma espontánea, logrando generar aprendizajes más significativos, facilitando el desarrollo de habilidades cognitivas, socioemocionales y físicas (Delgado \& López, 2013; Le Boulch, 1981; Morales \& Zabdy, 2017; Torres, 2002; UNICEF, 2018). Conjuntamente, jugar es una actividad intrínseca que responde a las necesidades de interacción, de estimulación, de socialización y de mejores procesos de aprendizaje que corresponden a la formación integral de los niños y las niñas durante la etapa preescolar (Zabalza Beraza, 2016).

Durante la etapa preescolar, los niños y niñas desarrollan sus habilidades cognitivas de acuerdo al crecimiento y maduración biológica, si a esto añadimos que el juego es la actividad predominante en los años preescolares, es posible categorizar como un mediador que jalone el desarrollo cognitivo de los niños, en definitiva, considerado como una de las actividades centrales en la vida de los infantes (Duncan \& Tarulli, 2003; Vygotsky, 1995) por lo tanto, generar espacios de aprendizaje que consideren tanto los objetivos descritos en el currículo, como las características propias vinculadas durante la etapa preescolar relacionadas a las $\mathrm{FE}$, serán imprescindibles para un óptimo desarrollo.

Parlebas (2008) presenta una categorización de los juegos psicomotores y juegos sociomotores, el primero tiene tres subniveles de clasificación, juegos psicomotores institucionalizados, los juegos psicomotrices tradicionales lúdicos $y$ los juegos libres. Los juegos psicomotores institucionalizados están asociadas a la resolución de tareas operatorias, estables y estandarizadas realizadas en solitario. Los juegos tradicionales de carácter lúdico, correspondiente a juegos tradicionales como juegos de pelota, de salto a la comba, rayuela, o donde simplemente se debe superar una serie de obstáculos establecidos en un circuito. Y, por último, los juegos libres, realizados en el patio de recreo, en un parque o entorno con contacto directo con la naturaleza; aquí el niño o niña 
no recibe una instrucción u orientación de un adulto, considerado de gran importancia, porque es el dueño y el gran responsable de llevar las riendas, teniendo una gran motivación interna y generando problemas que los mismos tienen que resolver, además de propiciar la autorregulación y la inhibición en conflictos que se le presentan y que los llevan a cambiar comportamientos impulsivos por mejores conductas, la expresión de sus emociones, el apoyo al bienestar social, la responsabilidad y el aprendizaje de normas también son conocidos, como juegos informales no institucionalizados. Por otro lado, los juegos sociomotrices, caracterizados por la presencia de una interacción motriz concreta en una comunicación motriz de tipo cooperativo y/o contracomunicación con finalidad antagónica. Por otro lado, Bueno (2019) los clasifica en juegos psicomotrices libres y juegos psicomotrices guiados, estos últimos, hacen referencia a actividades que organiza y coordina un adulto, y los niños y niñas están sujetos a las indicaciones de los mismos.

\section{METODOLOGÍA}

Se ha sugerido que la práctica de juegos motores y la optimización de las FE está relacionada con mejores procesos de aprendizaje en los planes de estudio y programas curriculares en niños y niñas durante la etapa infantil. Con el fin de examinar sistemáticamente esta hipótesis, se realizó una revisión sistemática para identificar los efectos producidos a nivel cognitivo y educativo. Para tal revisión, se plantea la metodología de investigación basada en los protocolos de búsqueda propuesto por Betancourt et al (2020) quiene dividen la búsqueda en dos protocolos, el primero es orientado en cinco aspectos tales como, el idioma de las fuentes de información, periodo de tiempo, las palabras clave, recursos de información y estrategias de búsqueda. El segundo protocolo, se estructuró en criterios de inclusión representados resumidos en la Tabla 1.

Tabla 1

Protocolo de búsqueda inicial

\begin{tabular}{|c|c|c|}
\hline Idioma & & Español - inglés - portugués - alemán \\
\hline Periodo de tiempo & & $2011-2021$ \\
\hline \multirow[t]{2}{*}{ Palabras claves| } & Individual & $\begin{array}{l}\text { Juego, funciones, ejecutivas, niños, desarrollo, cognitivo, } \\
\text { preescolares. } \\
\text { Play, executive, functions, cognitive, children, development. }\end{array}$ \\
\hline & Combinadas & $\begin{array}{l}\text { Juego motor, funciones ejecutivas, desarrollo cognitivo. } \\
\text { Motor play, executive functions, cognitive development. }\end{array}$ \\
\hline $\begin{array}{l}\text { Recursos de } \\
\text { información }\end{array}$ & Bases de datos & $\begin{array}{l}\text { Pubmed, Scopus, Spordiscus, Dialnet, Scielo, Google } \\
\text { Académico. } \\
\text { Revistas indexadas en formato digital. } \\
\text { Tesis de posgrados }\end{array}$ \\
\hline \multirow[t]{3}{*}{ Estrategias } & De formación & $\begin{array}{c}\text { Capacitación en uso de base de datos en función al tema en } \\
\text { cuestión }\end{array}$ \\
\hline & $\begin{array}{l}\text { De generación } \\
\text { de términos }\end{array}$ & $\begin{array}{c}\text { Combinación de bases de datos, revistas indexadas y } \\
\text { científicas. }\end{array}$ \\
\hline & De búsqueda & $\begin{array}{l}\text { 1. Búsqueda de términos } 2 \text {. Búsquedas booleanas } 3 \text {. } \\
\text { Consulta a páginas web } 4 \text {. Búsqueda con referencia a otras } \\
\text { investigaciones }\end{array}$ \\
\hline
\end{tabular}

Fuente: Los autores

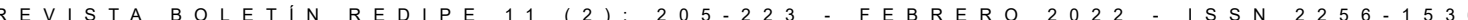


Los tópicos relevantes a la investigación y la estrategia de extracción de datos correspondientes al tema de estudio, como el título, el resumen y la introducción. De igual forma, la metodología Prisma, descrita en la figura 1, configuró la correspondencia entre las fuentes bibliográficas en estudios de tipo educativo, psicológico y de neurociencia; sucesión de los hechos planteados en cada artículo, aprobación de al menos un par evaluador y, por último, el abordaje del diseño de investigación experimental con corte longitudinal.
En cuanto a los criterios de exclusión, los artículos que no están relacionadas con el tema específico del objeto de estudio, entre ellos, los videojuegos utilizados para mejorar las habilidades cognitivas, los juegos motrices utilizados en intervenciones para niños y niñas con trastornos cognitivos o enfermedades atípicas de salud mental; se omitieron estudios que valoraban el desarrollo atípico o discapacidad intelectual, edades superiores o inferiores a las etapas preescolares y finalmente, publicaciones que no se encuentren en revistas científicas indexadas. En conformidad, se muestran los artículos seleccionados en la figura 1.

Figura 1

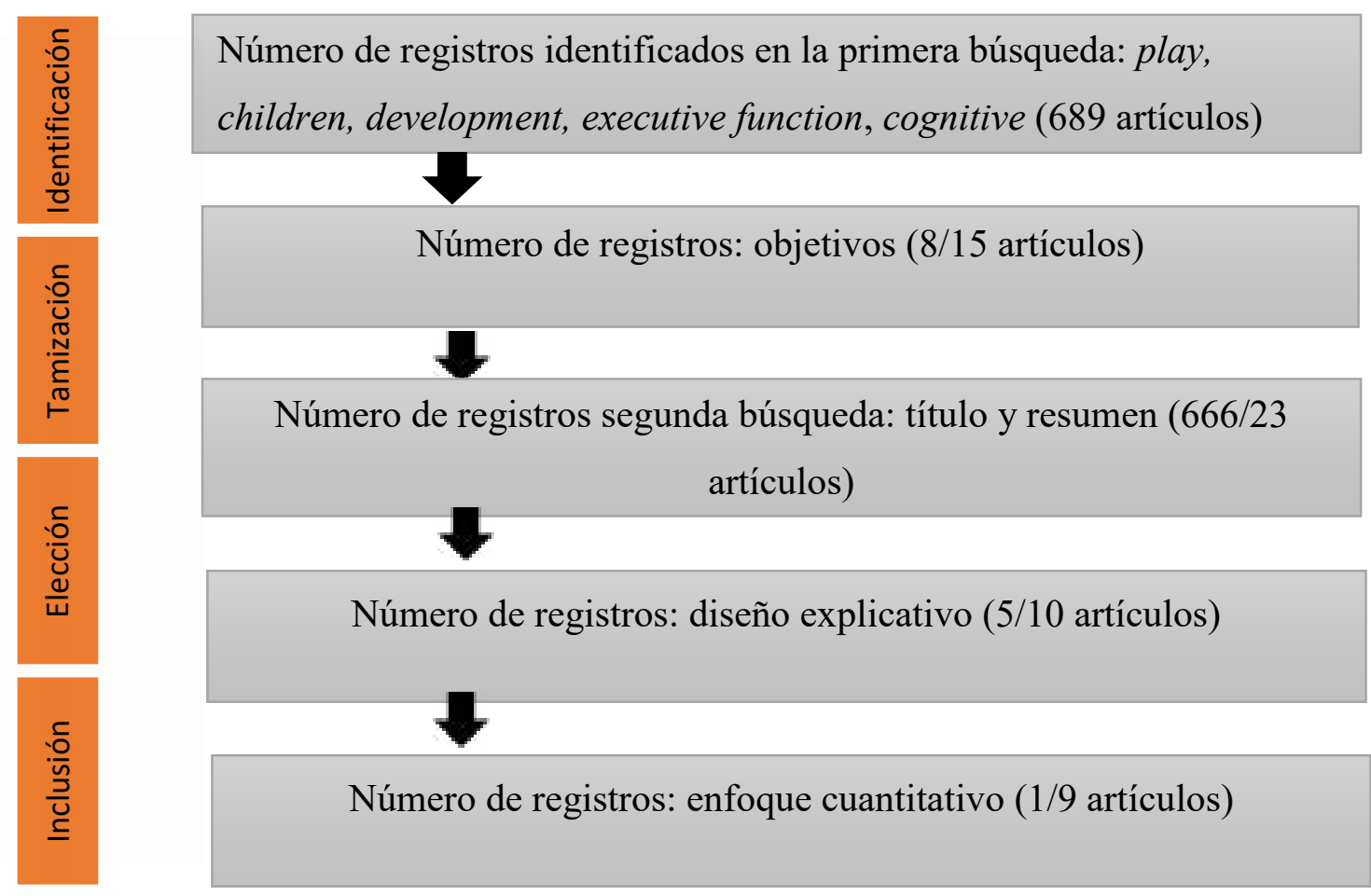

PRISMA Artículos seleccionados en diagrama de flujo.

\section{RESULTADOS}

Se incluyeron un total de nueve artículos, en la tabla 2 se reseña cada uno, iniciando con el autor, título, objetivo, diseño metodológico, muestra, instrumentos, resultados y las conclusiones obtenidas. Las muestras son homogéneas en cuanto al rango de edades para etapa preescolar, en cuanto al nivel socio económico no tuvo relevancia para su estudio; la clasificación de actividades implementadas, contenía juegos motrices que contemplaran la motricidad global o fina, las habilidades motrices básicas, juegos psicomotrices individuales y sociomotrices grupales, juegos de roles o simbólicos que incluyeran la coordinación dinámica global, 0 actividades motrices 
recreativas, además, fueron desarrolladas como procesos pedagógicos y didácticos de la educación física en contextos educativos y su incidencia en la optimización de las funciones ejecutivas durante la etapa preescolar.

Los estudios de Coelho et al. (2020); García et al. (2018); Muchiut, (2019); Röthlisberger et al. (2011) enfocaron sus investigaciones en la creación de planes y programas curriculares basados en juegos en el desarrollo de habilidades tanto motrices y sociales, y su influencia en las FE, logrando en estos casos, hacer las intervenciones en contextos educativos y su repercusión en el aprendizaje. Las pruebas seleccionadas con base en su validez neuropsicológica, asocian los cambios producidos en el proceso cognitivo.

Asimismo, los estudios consideran el funcionamiento ejecutivo, antes y después de la intervención, evaluada a través de test de Prueba de Stroop, Escala de Desarrollo Merril Palmer Revisada, test NEPSY II, cuestionario de madurez neuropsicológica infantil, Batería de evaluación del movimiento para niños-2, prueba de competencia matemática básica, etnografía visual, cuestionario de habilidades sociales dirigido a padres de familia, Índice de Procesamiento Controlado Numérico (NCPR), Índice de aleatoriedad numérica (RNG), una evaluación digital, Batería neuropsicológica para preescolares (BANPE) y Corazones y Flórez, CLASS Pre-K, Test de Forward Digit Span., tareas descriptivas, envoltura de regalo, tareas de dibujo circular, tareas de Stroop en computadoras, tarjetas dimensionales y tareas de trazado de senderos. En este orden de ideas, la más utilizada, fue la de Stroop.

En cuanto a la evaluación de los constructos de las FE, los estudios difieren, documentando la eficacia de sus intervenciones sólo en un conjunto limitado de FE. En correspondencia, los estudios de García et al. (2018), Traverso et al. (2015), Pesce et al. (2016) coincidieron en la valoración del control inhibitorio. De la misma forma, Amani et al. (2019) valoraron el control inhibitorio, la autorregulación y la flexibilidad cognitiva, por otro lado, Muchiut (2019) evaluaron la FE de planificación; por su parte, Rosas et al. (2019) estimaron el control inhibitorio, memoria de trabajo y flexibilidad cognitiva, asimismo, Thibodeau et al. (2016) midieron la memoria de trabajo, finalmente, Röthlisberger et al. (2011) apreciaron la memoria de trabajo, control Inhibitorio y flexibilidad Cognitiva. El único estudio de intervención que midió todas las FE fue realizado por (Coelho et al. 2020).

Ahora bien, los rangos de intervención de los estudios, oscilaban entre un mes y 6 meses aproximadamente; de una hora, hasta 3 horas distribuidas semanalmente; es decir, la duración a corto plazo frente a intervenciones a largo plazo, en cuanto a las metodologías de enseñanza se centraron en la resolución de problemas y la asignación de tareas; las evaluaciones de los programas de intervención incluían cuestionarios dirigidos a los padres de familia y docentes, consiguiendo resultados poco eficientes, al tener componentes subjetivos en las respuestas. En definitiva, se presenta en la tabla 2 la síntesis de resultados, respondiendo en forma independiente a la pregunta de investigación, y analizando el aporte de cada uno de los estudios.

\section{DISCUSIÓN}

El principal objetivo de esta investigación, consistió en poner de manifiesto la incidencia de los juegos motrices en el desarrollo de las FE como procesos pedagógicos y didáctico de la educación física durante etapas preescolares, así como también, identificar el impacto sobre la cognición en contexto educativos. Distintos tipos de juegos pueden apoyar el desarrollo de las FE, existe evidencia que vincula las intervenciones derivadas de los juegos motores pueden mejorar el control inhibitorio y la atención en niños con sobrepeso (Chin Chon, 2020). Por otra 
parte, el juego de tipo simbólico, tiene efectos significativos de aprendizaje, concernientes a la memoria de trabajo, siendo una herramienta que sirve para el desarrollo de la regulación emocional y el lenguaje, ambos considerados como precursores de las FE (Thibodeau, 2016).

Los juegos grupales o sociomotrices fortalecen las dimensiones de las FE, como la respuesta inhibitoria, la autorregulación y la flexibilidad cognitiva, ya que se crean mayores sinapsis entre las estructuras del sistema límbico y la neocorteza, correspondiente a mayor control y regulación de las emociones y conductas apropiadas en contextos de interacción social y educativo (Amani, 2019). Se deduce, que, en aquellos juegos motrices grupales, que requiere una interacción social y de cooperación, mostró efectos más fuertes en la promoción de la FE en comparación con juegos motrices en solitario, como también, los juegos motores que implican habilidades motoras de manipulación o juegos con pelota (Pesce et al. 2016).

Del mismo modo, los estudios que basan sus programas curriculares con enfoques cognitivos basados en el juego para la formación e integración de un adecuado funcionamiento ejecutivo, concluyeron centrarse en diseños metodológicos lúdicos, implementados en las etapas preescolares; evidenciando la eficacia de las actividades que requieran juegos motores en la promoción en el control inhibitorio, la memoria de trabajo y la flexibilidad cognitivo, teniendo mayor influencia en el aprendizaje de matemática y lenguaje en edades posteriores (Coelho, 2020; Traverso, 2015; Röthlisberger, 2011).

Igualmente, Coelho et al. (2020), demostró en su plan de estudios, el desarrollo de las FE basado en diversos tipos de juego, tales como, juegos simbólicos, rondas y de coordinación fina y que, además, puede incidir en el desarrollo de habilidades sociales y cognitivas, que, a su vez, permitirá que los niños tengan mayor control emocional, resolución de problemas, toma de decisiones, cualidades comunicativas y por ende, un mayor éxito en la escuela y a nivel profesional.

Por otro lado, distintos estudios usaron juegos de computadora como corazones y flores y Stroop para valorar la memoria, la atención y el control inhibitorio, y finalmente algunos estudios aplicaron baterías neuropsicológicas, siendo métodos más eficientes por su componente lúdico en la valoración de las FE en niños y niñas en etapas preescolares. Del mismo modo, se encontró diferencias en los entornos y aplicación de juegos motores, y que estos pueden dar lugar a diversas interpretaciones en diferentes dominios de FE, Por ejemplo, los juegos motrices complejos, que requiere una cognición controlada y una interacción social de cooperación, mostró efectos más fuertes en la promoción de la FE en comparación con juegos motrices en solitario y coordinación fina o simples (Amani, et al. 2019; Coelho, et al. 2020; Pesce, et al., 2016; Rosas, Espinoza et al. 2021; Röthlisberger et al. 2011; Thibodeau, et al. 2016; Traverso et al. 2015).

Continuando, es difícil comparar la incidencia de estas intervenciones utilizando un enfoque de causas-efectos, porque difieren en duración a largo plazo frente a intervenciones a corto plazo; la clasificación de juegos utilizados, como los juegos motrices individuales y juegos motores sociomotrices (grupales), como también juegos de motricidad global, o enfocados a la motricidad fina, pudieron influir en los resultados. Por último, los instrumentos de evaluación para medir las FE, se limitaron a medir entre una o tres FE, documentando la eficacia de las intervenciones sólo en un conjunto limitado de FE. Por lo tanto, se aluden a algunas limitaciones, al omitir variables que encarecen los programas y condicionan su viabilidad.

En consonancia, las FE tempranas predicen el desarrollo de precursores específicos de 
dominio del rendimiento académico, es decir, matemáticas y alfabetización (Traverso,2015; Roebers et al.,2011), lo que coloca a los niños con FE bien desarrolladas en una ventaja adicional. Los hallazgos subrayan la necesidad de implementar actividades o programas preescolares con el objetivo de fomentar el desarrollo de la $\mathrm{FE}$, idealmente en entornos institucionales. Por otro lado, se demuestra el vínculo concomitante entre juego motor y $\mathrm{FE}$, pues es precisamente en el movimiento y su secuencialización, donde el niño debe regular y controlar la acción motriz, dando origen a procesos cognitivos, estimulando diferentes áreas del cerebro, entre ellas, el sistema límbico y la neocorteza (Bueno i Torres, 2019; Guillen, 2017; Weineck, 2005)

Tabla 2. Características generales de artículos seleccionados

\begin{tabular}{|c|c|c|c|c|c|c|}
\hline Autor y Título & Objetivo & Metodología & Muestra & Instrumento & Resultados & Conclusiones \\
\hline $\begin{array}{l}\text { (García et al. } \\
2018 \text { ) Impacto } \\
\text { de un programa } \\
\text { de actividad } \\
\text { motriz con } \\
\text { funciones } \\
\text { ejecutivas } \\
\text { para el } \\
\text { fortalecimiento } \\
\text { del desarrollo } \\
\text { integral del } \\
\text { niño. }\end{array}$ & $\begin{array}{l}\text { Analizar el } \\
\text { impacto de } \\
\text { un programa } \\
\text { de actividad } \\
\text { motriz vinculado } \\
\text { con funciones } \\
\text { ejecutivas, en } \\
\text { desarrollo de } \\
\text { la cognición, } \\
\text { funciones } \\
\text { ejecutivas, } \\
\text { motricidad, } \\
\text { lectura, escritura } \\
\text { y matemáticas }\end{array}$ & $\begin{array}{l}\text { Experimental con } \\
\text { corte longitudinal } \\
\text { durante } 6 \text { meses. }\end{array}$ & $\begin{array}{l}66 \text { niños y } \\
\text { niñas entre } \\
5 \text { a } 7 \text { años } \\
\text { (35 grupo } \\
\text { experimental } \\
\text { y } 31 \text { grupo } \\
\text { control) }\end{array}$ & $\begin{array}{l}\text { Escala de } \\
\text { Desarrollo Merril } \\
\text { Palmer Revisada, } \\
\text { Test NEPSY II, } \\
\text { Cuestionario } \\
\text { de Madurez, } \\
\text { Neuropsicológica } \\
\text { Infantil, Batería } \\
\text { de evaluación } \\
\text { del movimiento } \\
\text { para niños-2 y } \\
\text { la prueba de } \\
\text { Competencia } \\
\text { Matemática } \\
\text { Básica }\end{array}$ & $\begin{array}{l}\text { F. Atencional } \\
=<\mathrm{F}=5.593 \\
p=0.024) . \\
\text { CI Control } \\
\text { Inhibitorio= } \\
\mathrm{F}=14.215 \\
p=0.001\end{array}$ & $\begin{array}{l}\text { Enfatizan la } \\
\text { importancia de } \\
\text { crear programas } \\
\text { motrices } \\
\text { vinculados con } \\
\text { las funciones } \\
\text { ejecutivas con } \\
\text { la finalidad de } \\
\text { fortalecer la } \\
\text { formación integral } \\
\text { del niño. }\end{array}$ \\
\hline $\begin{array}{l}\text { (Coelho et al. } \\
\text { 2020) } \\
\text { Desarrollo } \\
\text { de la función } \\
\text { ejecutiva en } \\
\text { niños en edad } \\
\text { preescolar } \\
\text { a través del } \\
\text { juego: un plan } \\
\text { de estudios }\end{array}$ & $\begin{array}{l}\text { Evaluar el plan } \\
\text { el estudio de } \\
\text { aprendizaje } \\
\text { BBF (Building } \\
\text { Brains and } \\
\text { Futures) basado } \\
\text { en juegos en el } \\
\text { mejoramiento de } \\
\text { las FE }\end{array}$ & $\begin{array}{l}\text { Tipo descriptiva } \\
\text { con enfoque } \\
\text { mixto, con } \\
\text { alcance } \\
\text { cuasiexperimental } \\
\text { de pre-prueba y } \\
\text { post prueba y con } \\
\text { corte longitudinal. }\end{array}$ & $\begin{array}{l}\text { Se intervinieron } \\
86 \text { niños } \\
\text { y niñas, } \\
72 \text { grupos } \\
\text { experimental y } \\
14 \text { en el grupo } \\
\text { control; en un } \\
\text { promedio de } \\
\text { edad entre } 3 \text { y } \\
6 \text { años. }\end{array}$ & Etnografía visual & $\begin{array}{l}\text { Valor } p<0,05= \\
0,02 \text { en las FE }\end{array}$ & $\begin{array}{l}\text { Proponen } \\
\text { implementar el } \\
\text { plan de estudios } \\
\text { BBF en etapa } \\
\text { preescolar para } \\
\text { mejorar las FE } \\
\text { en los niños, que } \\
\text { podría resultar } \\
\text { en una menor } \\
\text { necesidad de } \\
\text { educación } \\
\text { especial, una } \\
\text { disminución del } \\
\text { comportamiento } \\
\text { antisocial y } \\
\text { diagnósticos de } \\
\text { trastornos de la } \\
\text { FE. }\end{array}$ \\
\hline
\end{tabular}




\begin{tabular}{|c|c|c|c|c|c|c|}
\hline $\begin{array}{l}\text { (Amani et al. } \\
\text { 2019) } \\
\text { El efecto del } \\
\text { fortalecimiento } \\
\text { de las funciones } \\
\text { ejecutivas } \\
\text { mediante } \\
\text { juegos de } \\
\text { grupo en las } \\
\text { habilidades } \\
\text { sociales de } \\
\text { los niños de } \\
\text { preescolar }\end{array}$ & $\begin{array}{l}\text { Investigar el } \\
\text { efecto del } \\
\text { fortalecimiento } \\
\text { de las funciones } \\
\text { ejecutivas a } \\
\text { través de juegos } \\
\text { grupales en } \\
\text { las habilidades } \\
\text { sociales de niños } \\
\text { preescolares }\end{array}$ & $\begin{array}{l}\text { Estudio } \\
\text { cuasiexperi- } \\
\text { mental con } \\
\text { grupos } \\
\text { experimental } \\
\text { y de control y } \\
\text { diseño pre test } \\
\text { y post test, con } \\
\text { un tiempo de } \\
\text { intervención de } \\
12 \text { sesiones } \\
\text { de } 45 \text { minutos. }\end{array}$ & $\begin{array}{l}30 \text { niños y } \\
\text { niñas en etapa } \\
\text { preescolar }\end{array}$ & $\begin{array}{l}\text { Cuestionario } \\
\text { de habilidades } \\
\text { sociales dirigido a } \\
\text { padres de familia }\end{array}$ & $\begin{array}{l}\text { Los } \\
\text { comportamientos } \\
\text { sociales tuvieron } \\
\text { una significancia } \\
p<0,05 \text {. }\end{array}$ & $\begin{array}{l}\text { El fortalecimiento } \\
\text { de las } \\
\text { dimensiones de } \\
\text { las funciones } \\
\text { ejecutivas, como } \\
\text { la respuesta } \\
\text { inhibitoria, la } \\
\text { autorregulación } \\
\text { y la flexibilidad } \\
\text { cognitiva, a } \\
\text { través de los } \\
\text { juegos en } \\
\text { equipo mejora } \\
\text { las habilidades } \\
\text { sociales en los } \\
\text { niños }\end{array}$ \\
\hline $\begin{array}{l}\text { (Pesce et al. } \\
\text { 2016) } \\
\text { El juego } \\
\text { psicomotriz } \\
\text { libre y guiado, } \\
\text { benefician } \\
\text { conjuntamente } \\
\text { el desarrollo } \\
\text { motor y } \\
\text { cognitivo: } \\
\text { efectos } \\
\text { mediados y } \\
\text { moderados. }\end{array}$ & $\begin{array}{l}\text { Explorar la } \\
\text { relación entre la } \\
\text { educación física } \\
\text { y la cognición, } \\
\text { examinar si la } \\
\text { intervención en } \\
\text { la coordinación } \\
\text { motora mejora } \\
\text { los aspectos } \\
\text { cognitivos. }\end{array}$ & $\begin{array}{l}\text { Tipo de estudio } \\
\text { experimental, con } \\
\text { corte longitudinal } \\
\text { durante } 6 \text { meses } \\
\text { de intervención. }\end{array}$ & $\begin{array}{l}\text { La muestra } \\
\text { final estuvo } \\
\text { compuesta } \\
\text { por } 460 \text { niños, } \\
\text { de } 5-6 \text { años } \\
\text { (163), 7-8 (144) } \\
\text { y 9-10 años } \\
\text { (153), con } 232 \\
\text { (115 hombres } \\
\text { y } 117 \text { mujeres) } \\
\text { pertenecientes } \\
\text { a las clases } \\
\text { asignadas a los } \\
\text { enriquecidos. } \\
\text { PE y } 228 \text { (115 } \\
\text { varones y } \\
113 \text { mujeres) } \\
\text { pertenecientes } \\
\text { a las clases } \\
\text { tradicionales } \\
\text { de EF. }\end{array}$ & $\begin{array}{l}\text { Índice de } \\
\text { procesamiento } \\
\text { controlado } \\
\text { numérico. } \\
\text { Índice de } \\
\text { aleatoriedad } \\
\text { numérica (RNG), } \\
\text { una evaluación } \\
\text { digital }\end{array}$ & $\begin{array}{l}\text { Control Inhibitorio } \\
=p>0,019(>0,5)\end{array}$ & $\begin{array}{l}\text { Se pudo } \\
\text { determinar que la } \\
\text { FE de inhibición } \\
\text { tuvo un cambio } \\
\text { significativo } \\
\text { relacionado } \\
\text { con un óptimo } \\
\text { desarrollo motor, } \\
\text { especialmente } \\
\text { en los estilos } \\
\text { de enseñanza } \\
\text { de resolución } \\
\text { de problemas, } \\
\text { descubrimiento } \\
\text { guiado y } \\
\text { movimientos } \\
\text { que impliquen } \\
\text { el manejo de } \\
\text { pelotas. }\end{array}$ \\
\hline $\begin{array}{l}\text { (Muchiut, 2019) } \\
\text { Juego de } \\
\text { roles y función } \\
\text { ejecutiva de } \\
\text { planificación en } \\
\text { niños de Nivel } \\
\text { Inicial }\end{array}$ & $\begin{array}{l}\text { Implementar } \\
\text { un programa } \\
\text { de juego de } \\
\text { roles sociales } \\
\text { dirigido a niños } \\
\text { y niñas de 3-6 } \\
\text { años en jardines } \\
\text { infantiles en el } \\
\text { desarrollo de la } \\
\text { función ejecutiva } \\
\text { de planificación }\end{array}$ & $\begin{array}{l}\text { Metodología } \\
\text { fue explicativa } \\
\text { de tipo } \\
\text { cuasiexperimen- } \\
\text { tal con corte } \\
\text { longitudinal, La } \\
\text { Intervención duro } \\
20 \text { sesiones de } 1 \\
\text { hora } 2 \text { veces por } \\
\text { semana }\end{array}$ & $\begin{array}{l}\text { La muestra } \\
\text { estuvo } \\
\text { conformada por } \\
17 \text { niños de } 5 \\
\text { años cada uno. }\end{array}$ & $\begin{array}{l}\text { Batería } \\
\text { neuropsicológica } \\
\text { para preescolares } \\
\text { (BANPE) }\end{array}$ & $\begin{array}{l}\text { Función Ejecutiva } \\
\text { de Planificación } \\
p<0.001 \text { Grupo } \\
\text { Control } p<0.492\end{array}$ & $\begin{array}{l}\text { Las prácticas } \\
\text { pedagógicas } \\
\text { basadas en los } \\
\text { juegos deben } \\
\text { ser parte en las } \\
\text { instituciones } \\
\text { educativas, } \\
\text { especialmente } \\
\text { aplicados a las } \\
\text { poblaciones } \\
\text { infantiles, ya } \\
\text { que permite } \\
\text { aprovechar las } \\
\text { etapas evolutivas } \\
\text { y de desarrollo } \\
\text { neuropsicológico }\end{array}$ \\
\hline
\end{tabular}




\begin{tabular}{|c|c|c|c|c|c|c|}
\hline $\begin{array}{l}\text { (Rosas et al. } \\
\text { 2019) } \\
\text { Las funciones } \\
\text { ejecutivas } \\
\text { pueden } \\
\text { mejorarse en } \\
\text { niños en edad } \\
\text { preescolar } \\
\text { mediante } \\
\text { el juego } \\
\text { sistemático } \\
\text { en entornos } \\
\text { educativos: } \\
\text { evidencia de } \\
\text { un estudio } \\
\text { longitudinal }\end{array}$ & $\begin{array}{l}\text { Probar el } \\
\text { impacto del } \\
\text { juego en el } \\
\text { desarrollo } \\
\text { de funciones } \\
\text { ejecutivas (FE) } \\
\text { en niños en edad } \\
\text { preescolar }\end{array}$ & 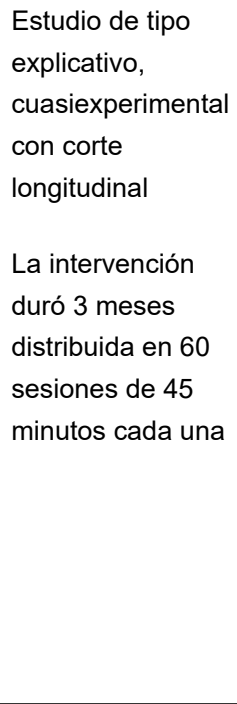 & $\begin{array}{l}\text { El estudio tuvo } \\
\text { una cantidad } \\
\text { de } 70 \text { niños y } \\
\text { niñas. }\end{array}$ & $\begin{array}{l}\text { Corazones y } \\
\text { Flórez. } \\
\text { CLASS Pre-K, }\end{array}$ & $\begin{array}{l}\text { FE de control } \\
\text { inhibitorio, } \\
\text { memoria } \\
\text { de trabajo y } \\
\text { flexibilidad } \\
\text { cognitiva con un } \\
\text { valor } p=<0.04 \\
(0.05)\end{array}$ & $\begin{array}{l}\text { Implementar en } \\
\text { los currículos } \\
\text { preescolares } \\
\text { actividades que } \\
\text { requieran juegos } \\
\text { y promover } \\
\text { las FE. Los } \\
\text { niños y niñas } \\
\text { con mejores } \\
\text { FE tienen } \\
\text { una influencia } \\
\text { mayor sobre el } \\
\text { aprendizaje en } \\
\text { matemáticas y } \\
\text { lenguaje. }\end{array}$ \\
\hline $\begin{array}{l}\text { (Thibodeau et } \\
\text { al. 2016) } \\
\text { Los efectos } \\
\text { del juego de } \\
\text { simulación } \\
\text { fantástico en el } \\
\text { desarrollo de } \\
\text { las funciones } \\
\text { ejecutivas: } \\
\text { un estudio de } \\
\text { intervención }\end{array}$ & $\begin{array}{l}\text { Determinar } \\
\text { experimental- } \\
\text { mente la } \\
\text { causalidad y } \\
\text { direccionalidad } \\
\text { de la relación } \\
\text { observada } \\
\text { entre Juegos } \\
\text { fantástico y FE } \\
\text { a través del } \\
\text { entrenamiento } \\
\text { del juego } \\
\text { orientado a } \\
\text { la fantasía en } \\
\text { niños en edad } \\
\text { preescolar }\end{array}$ & $\begin{array}{l}\text { Estudio de tipo } \\
\text { experimental con } \\
\text { corte longitudinal } \\
\text { durante } 5 \\
\text { semanas de } \\
\text { intervención }\end{array}$ & $\begin{array}{l}\text { El estudio tuvo } \\
\text { una muestra de } \\
110 \text { niños de } 3 \\
\text { y } 5 \text { años. }\end{array}$ & $\begin{array}{l}\begin{array}{l}\text { Cuestionarios y } \\
\text { entrevistas. }\end{array} \\
\text { Test de Forward } \\
\text { Digit Span. } \\
\text { Prueba de Stroop. }\end{array}$ & $\begin{array}{l}\text { La memoria de } \\
\text { trabajo MT } p=< \\
0.047\end{array}$ & $\begin{array}{l}\text { El juego de } \\
\text { simulación } \\
\text { fantástico durante } \\
\text { la infancia, como } \\
\text { un facilitador } \\
\text { de las FE. Los } \\
\text { resultados de } \\
\text { este estudio } \\
\text { indican que } \\
\text { fomentar el juego } \\
\text { de fantasía en la } \\
\text { vida cotidiana de } \\
\text { los niños facilitará } \\
\text { el desarrollo } \\
\text { en el dominio } \\
\text { cognitivo. }\end{array}$ \\
\hline $\begin{array}{l}\text { (Traverso et al. } \\
\text { 2015) } \\
\text { Mejora de } \\
\text { la función } \\
\text { ejecutiva en } \\
\text { la infancia: } \\
\text { evaluación } \\
\text { de una } \\
\text { intervención } \\
\text { formativa para } \\
\text { niños de } 5 \text { años }\end{array}$ & $\begin{array}{l}\text { Examinar la } \\
\text { eficacia de una } \\
\text { intervención } \\
\text { grupal para } \\
\text { niños de } 5 \text { años } \\
\text { que se centra en } \\
\text { los componentes } \\
\text { básicos de las } \\
\text { FE y evaluar } \\
\text { la eficacia de } \\
\text { un programa } \\
\text { de formación } \\
\text { diseñado para } \\
\text { promover la } \\
\text { FE durante } \\
\text { el período } \\
\text { preescolar, } \\
\text { particularmente } \\
\text { en niños de } \\
\text { cinco años }\end{array}$ & $\begin{array}{l}\text { Estudio de tipo } \\
\text { cuantitativo, con } \\
\text { corte longitudinal. } \\
12 \text { sesiones, } \\
\text { durante } 1 \text { mes. }\end{array}$ & $\begin{array}{l}\text { Niños y niñas } \\
\text { entre } 3 \text { y } 5 \\
\text { años de } 32 \\
\text { niños y } 43 \\
\text { niños con pre y } \\
\text { post test. }\end{array}$ & $\begin{array}{l}\text { Tareas } \\
\text { descriptivas } \\
\text { Envoltura de } \\
\text { regalo. }\end{array}$ & $\begin{array}{l}\text { Valor } p<0.05 \text { y } \\
p<0.01\end{array}$ & $\begin{array}{l}\text { El grupo de } \\
\text { entrenamiento } \\
\text { exhibió mayores } \\
\text { habilidades de } \\
\text { inhibición en } \\
\text { el control de } \\
\text { las respuestas } \\
\text { motoras según } \\
\text { lo medido por la } \\
\text { Tarea de dibujo } \\
\text { circular y en } \\
\text { el control de } \\
\text { las reacciones } \\
\text { impulsivas según } \\
\text { lo medido por } \\
\text { la Tarea de } \\
\text { figura familiar de } \\
\text { emparejamiento } \\
\text { preescolar. }\end{array}$ \\
\hline
\end{tabular}




\begin{tabular}{|c|c|c|c|c|c|c|}
\hline $\begin{array}{l}\text { (Röthlisberger } \\
\text { et al. 2011) } \\
\text { Mejora de las } \\
\text { FE en niños de } \\
5 \text { y } 6 \text { años. }\end{array}$ & $\begin{array}{l}\text { Investigar los } \\
\text { efectos de un } \\
\text { entrenamiento } \\
\text { basado en un } \\
\text { plan de juegos } \\
\text { en grupos } \\
\text { pequeños } \\
\text { que se centra } \\
\text { en diferentes } \\
\text { componentes de } \\
\text { los FE en niños } \\
\text { de prekinder y } \\
\text { kindergarten. }\end{array}$ & $\begin{array}{l}\text { Estudio } \\
\text { cuantitativo, con } \\
\text { corte longitudinal } \\
\text { con una } \\
\text { intervención de } \\
\text { con grupo control } \\
\text { y experimental, } \\
6 \text { semanas en } \\
30 \text { sesiones en } \\
\text { niños y niñas de } \\
\text { preescolares. }\end{array}$ & $\begin{array}{l}\text { Participaron } \\
135 \text { niños de } \\
5 \text { y } 6 \text { años de } \\
\text { edad. }\end{array}$ & $\begin{array}{l}\text { Tarjetas } \\
\text { dimensionales y } \\
\text { Stroop. }\end{array}$ & $\begin{array}{l}\text { Memoria de } \\
\text { Trabajo. } p< \\
0.05 \text {. Control } \\
\text { Inhibitorio. } \\
p<0.01 . \\
\text { Flexibilidad } \\
\text { Cognitiva } p<0.01\end{array}$ & $\begin{array}{l}\text { Los niños en } \\
\text { edad preescolar } \\
\text { mejoraron } \\
\text { sustancialmente } \\
\text { su memoria } \\
\text { de trabajo y } \\
\text { los procesos } \\
\text { de flexibilidad } \\
\text { cognitiva, } \\
\text { mientras que } \\
\text { se encontraron } \\
\text { efectos de } \\
\text { entrenamiento } \\
\text { significativos } \\
\text { para los niños de } \\
\text { jardín de infancia } \\
\text { en el control } \\
\text { inhibitorio. }\end{array}$ \\
\hline
\end{tabular}

Fuente: Los autores.

\section{CONCLUSIONES}

La literatura indica que el FE como la flexibilidad cognitiva, la memoria de trabajo, el control inhibitorio y la planificación, se fomentan practicando actividades recreativas con un componente motriz global. Por supuesto, otras actividades realizadas por los niños, como tocar un instrumento musical, y de artes plásticas tienen efectos igualmente significativos sobre las FE, pero no fueron sujetos a análisis durante este estudio.

Igualmente, se ha relacionado el aumento de las FE derivado de la práctica de juegos motores de moderada o vigorosa intensidad, con los beneficios que representa a nivel cognitivo, es decir, los juegos motores que involucran la contracción de grandes grupos musculares, movilizan las células musculares que generan Irisina, lo que incrementa la producción el Factor Derivado del Cerebro (BDNF), neurotransmisor activador de la plasticidad sináptica, incrementando de este modo la cognición, el aprendizaje y la memoria (Bueno, 2019; Huber et al. 2007; Gordon et al. 2003; Gordon et al. 2002).
Por lo tanto, es imprescindible tener en cuenta, para que haya tales cambios en los niños y niñas que cursan la etapa preescolar, deben realizar actividades que genere la motricidad global o la ejecución de habilidades motrices más complejas que requieran coordinación global, pues la mayoría de estudios fueron limitados a juegos motores de coordinación fina, es decir, los estímulos involucran la regulación y control de acciones motrices de pocos grupos musculares, haciendo salvedad de juegos que implicarán la asociación de movimientos coordinados y regulados globalmente.

No obstante, se demostró la importancia de usar los juegos como medio de aprendizaje, de allí la importancia de incluir actividades lúdicas estructuradas, enfocadas al juego motor y su relación directa con el desarrollo cognitivo. En este sentido, los resultados sugieren que vale la pena utilizar los juegos motores como medio de aprendizaje durante la etapa preescolar, porque influye positivamente en las FE en el desarrollo de programas basados en juegos motores que impliquen aspectos motrices coordinativos, destinados a mejorar las FE, ya que, la educación infantil es un periodo sensible de neuromaduración, puesto que se establecen 
mayor cantidad de conexiones neuronales, que van a constituir la base del aprendizaje y la conducta (García et al. 2018; Weineck, 2005) y sentarán fundamentos en la adquisición de aprendizajes más complejos, como la aritmética y el castellano.

En síntesis, los juegos motores tienen un elemento vital a través del movimiento, es decir, "moverse mientras juega y mientras juega, aprende", y esas capacidades motrices que dan origen a procesos cognitivos, no dependen solamente del aprendizaje de un movimiento, también dependen de él, los sentimientos, las emociones y el comportamiento social, por lo tanto, están supeditadas a la integración en los estímulos a nivel cognitivo y emocional, por ende, es necesario utilizar métodos de enseñanza-aprendizaje adecuados, que en clave educativa, implique en estas edades una motivación sensorial, para sentar bases de desarrollo posterior, siendo imprescindible una estimulación concurrente de programas curriculares, en los que sea visible el rol que cumplen los docentes, en la necesidad de mejorar la construcción de intervenciones identificando acciones en pro del desarrollo integral durante etapas preescolares, donde se debe incluir una pedagógia y didáctica que favorezca el desarrollo motor y propicie dentro de los planes de estudio, el perfeccionamiento de competencias motrices, emocionales y cognitivas que a su vez, permitan una formación integral a través del juego y el movimiento.

\section{REFERENCIAS BIBLIOGRÁFICAS}

Alesi, M., Giordano, G., Giaccone, M., Basile, M., Costa, S., \& Bianco, A. (2020). Effects of the Enriched Sports ActivitiesProgram on Executive Functions in Italian Children. Journal of Functional Morphology and Kinesiology, 5(2), 26. doi: https://doi.org/10.3390/ jfmk5020026
Amani, M., Koruzhdeh, E., \& Taiyari, S. (2019). The Effect of Strengthening Executive Functions Through Group Games on the Social Skills of Preschool Children. Games Health J, 8(3), 213-219. doi:doi: 10.1089/g4h.2018.0052.

Barkley, R. A. (2011). ¿Es el funcionamiento ejecutivo deficiente en el TDAH? Depende de sus definiciones y sus medidas. The ADHD Report, 19(4), 1-9. doi:https://doi.org/10.1521/ adhd.2011.19.4.1

Bartholomew, J., Jowers, E., Errisuriz, V., Vaughn, S., \& Roberts, G. (2017). A Cluster randomized control trial to assess the impact of active learning on child activity, attention control, and academic outcomes: The Texas I-CAN trial. Randomized Controlled Trial, 61, 81-86. doi:https://doi.org/10.1016/j. cct.2017.07.023

Bausela Herreras, E. (2014). Funciones ejecutivas: nociones del desarrollo desde una perspectiva neuropsicológica. Acción Psicológica, 11(1), 21-34. Obtenido de https://scielo.isciii.es/scielo. php?script=sci_arttext $\&$ pid $=$ S1578908X2014000100003

Betancourt, M., Bernate, J., Fonseca, I., \& Rodriguez, L. (2020). Revisión documental de estrategias pedagógicas utilizadas en el área de la educación física, para fortalecer las competencias ciudadanas. Retos, 38(38), 845-851. doi:https://doi.org/10.47197/retos. v38i38.74918 
Blair, C., \& Raver, C. (2015). School Readiness and Self-Regulation: A Developmental Psychobiological Approach. Department of Applied Psychology, 66(1), 711-731. doi:https://doi.org/10.1146/annurevpsych-010814-015221

Braidot, N. (2015). Mejora tu agilidad mental. Barcelona: Centro libros PAPF.

Bueno i Torres, D. (2019). Neurociencia aplicada a la Educación. Madrid: SINTESIS.

Cameron, C., Murrah, W., Grissmer, D., Brock , L., Bell, L., \& Morrison, F. (2012). Fine Motor Skills and Executive Function Both Contrubute to Kindergarten Achievement. Child Development, 83(4), 1129-1244. doi:10.1111/j.14678624.2012.01768.x

Chin Chou, C., Chou Chen, K., Yao Huang, M., \& Yu Tu, H. (2020). Can Movement Games Enhance Executive Function in Overweight Children? A Randomized Controlled Trial. Journal of Teaching in Physical Education, 39(4), 527-535. doi:https://doi.org/10.1123/jtpe.20190165

Coelho, L., Amatto, A., Gonzalez, C., \& Gibb, R. (2020). Building executive function in pre-school children through play: a curriculum. International Journal of Play, 9(11), 128-142. doi:10.1080/21594937. 2020.1720127

Delgado Sotelo, A., \& Lopez Imbacuan, E. (2013). El juego como generador de aprendizaje. Revista Criterios, 20(1), 203-218. Obtenido de http://editorial. umariana.edu.co/revistas/index.php/ Criterios/article/view/1863

Diamond, A., \& Ling , D. (2016). Conclusions about interventions, programs, and approaches for improving executive functions that appear justified and those that, despite much hype, do not. Developmental Cognitive Neuroscience, 18,34-48. doi:http://dx.doi.org/10.1016/j. dcn.2015.11.005

Diaz, A. e. (1993). Desarrollo Curricular para la formación de maestros especialistas en Educación Física. Editorial Gymnos.

Duncan, R., \& Donato, T. (2003). Play as the Leading Activity of the Preschool Period: Insights from Vygostsky, Leont'ev, and Bakhtin. Early Education and Development, 14(3), 271-292. doi:10.1207/s15566935eed1403_2

García Fernández, D., Chanez Valenzuela, M., Cruz Chávez, C., Gueda Delgado, J., Velázquez Saucedo, G., \& Zubiair González, M. (01 de 2018). Impacto de un programa de actividad motriz con funciones ejecutivas para el fortalecimiento del desarrollo integral del niño. Scientific Technical Journal of School Sport, 4(1), 37-58. Obtenido de https://dialnet.unirioja.es/metricas/ documentos/ARTREV/6262917

Goh, T. L., Hannon, J., Webster, C., \& Podlog, L. (2017). Classroom teachers' experiences implementing a movement integration program: Barriers, facilitators, and continuance. Teaching and Teacher Education, 66, 88-95. doi:https://doi. org/10.1016/j.tate.2017.04.003

Gonzáles Osornio, M. (2014). Desarrollo Neuropsicológico de las Funciones Ejecutivas en la Edad Preescolar. El Manual Moderno.

Gordon, N. S., Burke, S., Akil, H., Watson, S. J., \& Panksepp, J. (2003). Socially-induced brain 'fertilization': play promotes brain derived neurotrophic factor transcription in the amygdala and dorsolateral 
frontal cortex in juvenile rats $2003 \mathrm{Apr}$ 24;341(1):17. Neurosci Lett, 341(1), 1720. Obtenido de https://doi.org/10.1016/ s0304-3940(03)00158-7

Gordon, N., Kollack-Walker, S., Akil, H., \& Panksepp, J. (2002). Expression of c-fos gene activation during rough and tumble play injuvenile rats. Brain Res Bull, 57(5), 651-659. doi:pmid:11927369

Guillen , J. (2017). Neuroeducación en el aula: De la teoría a la práctica. Spanish Edition.

Hernandez-Sampieri, R., \& Torres C, P. M. (2018). Metodología de la investigación (Vol. 4). México^ eD. F DF: McGraw-Hill Interamericana.

Huber , R., Tononi, G., \& Cirrelli, C. (2007). Exploratory behavior, cortical BDNF expression, and sleep homeostasis. Sleep, 30(2), 129-139. doi:pmid:17326538

Huizinga, J. (1990). Homo Ludens. Alianza.

Ivrendi, A. (2020). Early childhood teachers' roles in free play. Early Years, 40(3), 273-286. Obtenido de https://doi.org/10. 1080/09575146.2017.1403416

Johnson, J. E., Christie, J. F., Yawkey, T. D., \& Wardle, F. (1987). Play and early childhood development. Longman.

Le Boulch, J. (1981). El desarrollo psicomotor desde el nacimiento a los seis años. Doñate.

Lundy, A., \& Trawick-Smith, J. (2020). Effects of Active Outdoor Play on Preschool Children's on-Task Classroom Behavior. Early Childhood Education Journal volume, 49(1), 463-471. doi:10.1007/ s10643-020-01086-w
Merino, V. (Abril de 2016). A new vaccine: vaccine for self-knowledge. Neurobiological base of human behavior. The game between the instintive-emocional brain and the rational brain. Pediatr Aten Primaria, 18(70), 85-91. Obtenido de https://www.scopus.com/record/display. uri?eid $=2$

Mora, F. (2013). Neuroeducación. Alianza Editorial.

Mora, F. (2017). Neuroeducaión. Alianza Editorial.

Moriguchi, Y., \& Hiraki, K. (2011). Longitudinal development of prefrontal function during early childhood. Neurociencia Cognitiva del Desarrollo, 1(2), 153-162. doi:https:// doi.org/10.1016/j.den.2010.12.004

Muchiut, Á. F. (2019). Juego y función ejecutiva de planificación en niños de Nivel Inicial. Cuadernos de Neuropsicología / Panamerican Journal of Neuropsychology, 13(2), 163-170. Obtenido de https://www.redalyc.org/ journal/4396/439667351014/html/

Pesce, C., Masci, I., Marchetti, R., Vazou, S., Sääkslahti, A., \& Tomporowski, P. (2016). Deliberate Play and Preparation Jointly Benefit Motor and Cognitive Development: Mediated and Moderated Effects. Frontiers in Psychology, 7(349). doi:https://doi.org/10.3389/ fpsyg.2016.00349

Piaget, J. (1956). Motricité, percepción et intelligence. Enfance.

Ramos , D., Bianchi , M., Rabello, E., \& Martins, M. (2019). Intervenções com jogos em contexto educacional: Contribuições às funções executivas. Psicologia: Teoria e Prática, 21(2), 294-315. doi:10.5935/1980-6906/psicologia 
Rigal, R. (2006). Educación motriz y educación psicomotriz en preescolar y primaria. Inde.

Rosas , R., Espinoza, V., Porflitt, F., \& Ceric, F. (2019). Executive Functions Can Be Improved in Preschoolers Through Systematic Playing in Educational Settings: Evidence From a Longitudinal Study. Frontiers psychology. doi:10.3389 / fpsyg.2019.02024

Röthlisberger, M., Neuenschwander, R., Cimeli, P., Michel, E., \& Roebers, C. (2011). Improving executive functions in 5and 6-year-olds: Evaluation of a small group intervention in prekindergarten and kindergarten children. Infant and child development, 21(4), 411-429. doi:https://doi.org/10.1002/icd.752

Sarlé, P. (2011). El juego como espacio cultural imaginario y didáctico . Revista infancias imágenes, 10(2), 83-91. doi:https://doi. org/10.14483/16579089.4451

Sarlé, P. M. (2016). La inclusión del juego en las salas de educación infantil: espontaneidad o regulación. RELADEI - Revista latinoamericana de educación infantil, 5(2), 17-27. Recuperado el 2021 de abril de 28, de https://revistas.usc. gal/index.php/reladei/article/view/4913

Stein, A., Migdalek, K., \& Sarlé, P. (2012). "Te Enseño a Jugar": Caracterización de Movimientos Interaccionales y Formas Lingüísticas Mediante las Cuales se Regula la Interacción Lúdica. Psykhe, 21(1), 55-67. doi:http://dx.doi.org/10.4067/S071822282012000100004

Thibodeau, R., Gilpin, A., Brown, M., \& Meyer, B. (2016). The effects of fantastical pretend-play on the development of executive functions: An intervention study. Journal of Experimental Child Psychology, 145, 120-138. doi:https:// doi.org/10.1016/j.jecp.2016.01.001

Torres, C. (2002). El juego una estrategia importante. Educere, 6(19), 289-296. Obtenido de https://www.redalyc.org/ articulo.oa? $\mathrm{id}=35601907$

Traverso, L., Viterbori, P., \& Usai, M. C. (2015). Improving executive function in childhood: evaluation of a training intervention for 5-year-old children. Frontiers in Psychology, 6. doi:https:// doi.org/10.3389/fpsyg.2015.00525

Tsujimoto, S., Yamamoto, T., Kawaguchi, H., Koizumi, H., \& Sawaguchi, T. (14 de Julio de 2004). Prefrontal cortical activation associated with working memory in adults and preschool children: an eventrelated optical topography study. Clinica Trial, 7, 703. doi:10.1093/cercor/bhh030

UNICEF. (2018). Aprendizaje a través del juego. UNICEF Publicaciones. Recuperado el 27 de Abril de 2021, de https://www. unicef.org/sites/default/files/2019-01/ UNICEF-Lego-Foundation-Aprendizajea-traves-del-juego.pdf

Vidarte , J., \& Orozco , C. (2015). Relaciones entre el desarrollo psicomotor y el rendimiento académico en niños de 5 y 6 años de una institución educativa de La Virginia (Risaralda, Colombia). Revista Latinoamericana de Estudios Educativos, 11(2), 190204. Obtenido de http://vip.ucaldas. edu.co/latinoamericana/downloads/ Latinoamericana11(2)_9.pdf 
Vygotsky, L. (1995). Obras escogidas Tomo III. Madrid: Visor.

Weineck, J. (2005). Entrenamiento Total. Paidotribo.

Weinsten, Weinstein, A., Voss, M., Prakash, R., Chaddock, L., Szabo, A., \& White, S. (2011). The association between aerobic fitness and exutive function is mediated by prefrontal cortex volumen. Brain, Behavior, and Immunity, 26, 811819. doi:10.1016/j. bbi.2011.11.008

Yogman, M., Garner, A., Hutchinson , J., HirshPasek, K., \& Michnick-Golinkoff, R. (2018). The power of play: A pediatric role in enhancing development in young children. Pediatrics, 142(3), 1-18. doi:https://doi.org/10.1542/peds.20182058

Zabalza Beraza, M. Á. (2016). La felicidad de jugar. RELADEI - Revista latinoamericana de educación infantil, 5(2), 8-12.

Zelazo, P., Carsol, S., \& Kesek, A. (2008). The development of executive function in childhood. Developmental cognitive neuroscience. Handbook of developmental cognitive neuroscience. MIT Press. 